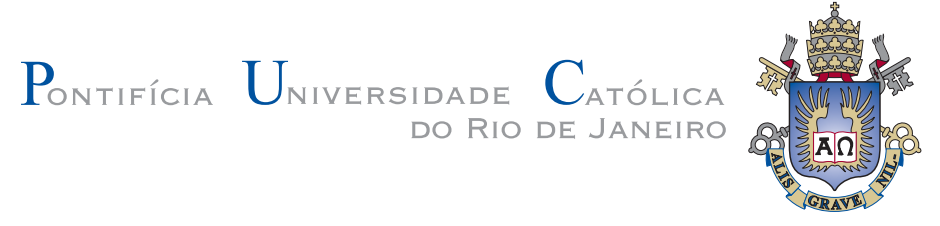

Júlio César da Silva

\title{
MODELAGEM E SIMULAÇÃO NUMÉRICA DO FLUXO BIFÁSICO E DO TRANSPORTE MULTICOMPONENTE EM MEIOS POROSOS COM INTERAÇÃ̃O TERMO-QUÍMICA
}

Tese apresentada ao Programa de Pós-graduação em Engenharia Civil do Departamento de Engenharia Civil da PUC-Rio como requisito parcial para obtenção do título de Doutor em Engenharia Civil Geotecnia.

Orientador: Prof. Eurípedes do Amaral Vargas Jr. 


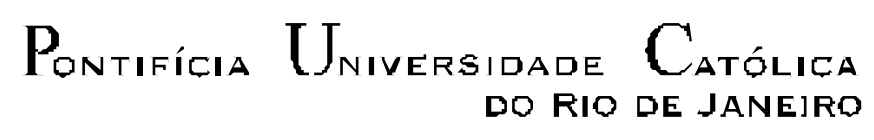

DO RIO DE JANE]RO

Júlio César da Silva

Modelagem e simulação numérica do fluxo bifásico e do transporte multicomponente em meios porosos com interação termo-química

Tese apresentada como requisito parcial para obtenção do título de Doutor pelo Programa de Pós-Graduação em Engenharia Civil da PUC-Rio. Aprovada pela Comissão Examinadora abaixo assinada.

Prof. Eurípedes do Amaral Vargas Jr. Presidente/Orientador Departamento de Engenharia Civil - PUC-Rio

Prof. Olavo Barbosa Filho Departamento de Ciência dos Materiais e Metalurgia- PUC-Rio

Prof. Tácio Mauro P. de Campos Departamento de Engenharia Civil - PUC-Rio

Dra. Andrea Ferreira Borges CENPES/Petrobras

Prof. Marcio Arab Murad

LNCC

Prof. José Eugênio Leal Coordenador Setorial do Centro Técnico Científico - PUC-Rio 
Todos os direitos reservados. É proibida a reprodução total ou parcial do trabalho sem autorização da universidade, do autor e do orientador.

\section{Júlio César da Silva}

Graduou-se em Engenharia Civil na EM/UFOP (Escola de Minas da Universidade Federal de Ouro Preto) em 1996, defendeu sua dissertação de mestrado em Engenharia Civil (Geotecnia) na PUC-Rio (Pontifícia Universidade Católica do Rio de Janeiro) em 1999 e, atualmente, trabalha como pesquisador pelo TecGraf/PUC-Rio.

Ficha Catalográfica

Silva, Júlio César da

Modelagem e simulação numérica do fluxo bifásico e do transporte multicomponente em meios porosos com interação termo-química/ Júlio César da Silva; orientador: Prof. Eurípedes do Amaral Vargas Jr.. - Rio de Janeiro : PUC-Rio, Departamento de Engenharia Civil, 2004.

v., 123 f: il. ; 29,7 cm

1. Tese (doutorado) - Pontifícia Universidade Católica do Rio de Janeiro, Departamento de Engenharia Civil.

Inclui referências bibliográficas.

1. Engenharia Civil - Teses. 2. Fluxo bifásico. 3. Transporte multicomponente. 4. Acoplamento químico. 5. Acoplamento térmico. 6. Oxidação química. 7. Método dos elementos finitos. 8. Meios porosos não saturados. I. Vargas Jr., Eurípedes do Amaral. II. Pontifícia Universidade Católica do Rio de Janeiro. Departamento de Engenharia Civil. III. Título. 


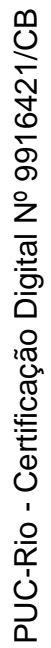

À minha pequena grande família. 


\section{Agradecimentos}

Ao professor Vargas, orientador deste trabalho, pelos grandes ensinamentos durante a discussão deste tema e pelo direcionamento do enfoque do mesmo.

Ao Ondra Sracek pelos ensinamentos na área de geoquímica e pelas discussões de alguns resultados obtidos neste trabalho.

A Andrea Borges pelas trocas de informações sobre a modelagem do fluxo bifásico e ao professor Olavo pelas discussões sobre a drenagem ácida de resíduos mineiros.

A minha pequena grande família (minha esposa Fabrícia e minha filhinha Lavínia) pelo amor, compreensão e incentivo nos momentos difíceis.

A meus pais (Geraldo e Consolada), meus irmãos (Fabinho, Claudinha, Lange e Cris), meus sobrinhos (Pedro, Yasmim e Gabriela), meus cunhados, a família de minha esposa (agora minha, também) e a todos meus parentes e amigos de Ouro Preto, que torceram por mais esta conquista.

A todos os integrantes da eterna família PS (atuais e "ex"), atualmente Paulinho, Eugênio, Leitinho e Alfredo.

A meus amigos de longas datas no Rio de Janeiro: Bodim, Eudes, Cleide, Cadu, Isabelle, Antonio Sérgio, Fred Leal, Vitor, Heber, Ataliba e Anna Paula.

Aos demais amigos do TecGraf, em especial, Ivan, Fred, Turco, Lula, Camilo, Anderson, Pasquetti, Ramires, Sandra, Herivelto e Claudinei.

A ANP pelo apoio financeiro.

A todos colegas da pós-graduação não mencionados aqui, professores e funcionários do Departamento de Engenharia Civil da PUC-Rio e do TecGraf, que contribuíram de forma direta ou indireta para realização deste trabalho.

Em especial, a DEUS. 


\section{Resumo}

Silva, Júlio César da; Vargas Jr., Eurípedes do Amaral. Modelagem e simulação numérica do fluxo bifásico e do transporte multicomponente em meios porosos com interação termo-química. Rio de Janeiro, 2004. 123p. Tese de Doutorado — Departamento de Engenharia Civil, Pontifícia Universidade Católica do Rio de Janeiro.

Este trabalho apresenta o estágio atual do desenvolvimento de uma ferramenta numérica para simulação e análise do fluxo bifásico e do transporte multicomponente e de calor em meios porosos com interação química em duas dimensões. O programa desenvolvido foi baseado no UNSATCHEM_2D escrito em linguagem Fortran. A discretização das equações diferenciais parciais é feita em elementos finitos no espaço e em diferenças finitas no tempo. Uma interface gráfica foi criada em linguagem Lua para a entrada dos dados e visualização dos resultados. Esta interface foi baseada no programa Mtool do TecGraf. O objetivo do trabalho foi implementar uma ferramenta que acoplasse diferentes processos em meios porosos: fluxo bifásico, transporte de solutos, processos químicos e temperatura. A ferramenta desenvolvida deverá ser útil em análises de problemas ambientais. Em particular, o enfoque deste trabalho é no acoplamento de processos químicos. Para isto, uma rotina de especiação química foi acoplada à cinética química com relevância na oxidação mineral. Para resolver o problema da especiação química, o programa Phreeqc foi utilizado. $\mathrm{O}$ trabalho apresenta detalhes dos processos de análise, de suas equações relevantes e da implementação numérica. No estágio atual de desenvolvimento, o programa é capaz de avaliar o transporte de metais e, em particular, a simulação da drenagem ácida em barragens de rejeito e pilhas de estéril sob condições complexas. Exemplos de validação e ilustrativos são mostrados e discutidos. São sugeridas algumas possibilidades de futuros acoplamentos à ferramenta numérica desenvolvida.

\section{Palavras-chave}

fluxo bifásico; transporte multicomponente; acoplamento químico; acoplamento térmico; oxidação química; método dos elementos finitos; meios porosos não saturados. 


\section{Abstract}

Silva, Júlio César da; Vargas Jr., Eurípedes do Amaral. Modelling and numerical simulation of the two phases flux and transport multicomponent in the porous medium with no isotermic-chemical interaction. Rio de Janeiro, 2004. 123p. D.Sc. Thesis — Departament of Civil Engineering, Pontifícia Universidade Católica do Rio de Janeiro.

This work presents the present stage of development of a numerical platform for simulation and analysis of two-phase flow and of, non isotermic, multicomponent transport in porous media with chemical interaction in two dimensions. The developed computer code is based on the UNSATCHEM_2D code written in Fortran language. The discretization of the partial differential equations is done by finite elements at the space and in finite difference at the time. A graphical interface was created in language Lua for the input of the data and result visualization. This interface was based on the program TecGraf's Mtool. The purpose of the work was to construct a numerical tool that couples different processes in porous media: flow (two-phase), transport of solutes, chemical processes and temperature. The developed tool should be useful in the analysis of environmental problems. In particular, the work focused on the coupling of chemical processes. For this, a chemical speciation routine was coupled to chemical kinetics with relevance to mineral oxidation. In order to solve the chemical speciation problem, the program Phreeqc was used. The work presents details of the analyzed processes, their relevant equations and of the numerical implementation. At the present stage of development, the computer program is able to evaluate the transport of metals and, at particular, the simulation of the acid mine drainage at tailings discharge and waste rock piles under complex conditions. Validation and illustrative examples are shown and discussed. Possibilities of future additions to the developed tool are suggested.

\section{Keywords}

flux two-phases; multicomponent transport; chemical interaction; non isotermic interaction; chemical oxidation; finite elements methods; unsaturated porous media. 


\section{Sumário}

1 Introdução 19

2 Fluxo em meios porosos $\quad 21$

2.1 Fluxo monofásico 21

2.2 Fluxo bifásico 22

3 Acoplamentos 28

3.1 Transporte de contaminantes em meios porosos 28

3.2 Acoplamento químico 35

3.3 Acoplamento térmico 43

3.4 Acoplamento biológico 47

3.5 Dissolução 50

4 Modelagem numérica $\quad 53$

4.1 Métodos numéricos 53

4.2 Métodos iterativos 56

4.3 Modelagem em elementos finitos e diferenças finitas 59

5 Descrição da ferramenta numérica $\quad \mathbf{7 2}$

5.1 Trabalho implementado $\quad 72$

5.2 Descrição do programa de análise 74

5.3 Interface gráfica 76

5.4 Relevância 78

5.5 Aplicações $\quad 78$

6 Exemplos $\quad \mathbf{8 4}$

6.1 Exemplo de validação $\quad 84$

6.2 Exemplos ilustrativos 96

7 Conclusões e sugestões 112

7.1 Conclusões 112

7.2 Sugestões para trabalhos futuros 113

$\begin{array}{lr}\text { Referências Bibliográficas } & 115\end{array}$ 


\section{Lista de Figuras}

2.1 Relação entre $B_{l}$ (para fase $\mathrm{NAPL}=B_{o}$ ), $B_{g}$ e $R_{s}$ com a pressão (modificado de Huyakorn e Pinder, 1983 [14]). O ponto onde o valor da pressão é igual à pressão de formação de bolhas $P=22700 k P a$ está indicado com um triângulo (Borges, 2002 [81]).

3.1 Acoplamentos para transporte multicomponente em diversas fases.

3.2 Diferentes tipos de comportamento de partículas sólidas em reação (Levenspiel, 1974 [8]).

3.3 Modelo de núcleo não reagido (Levenspiel, 1974 [8]).

4.1 Ilustração gráfica do método iterativo de Picard em uma variável (Huyakorn e Pinder, 1983 [14]).

4.2 Ilustração gráfica do método iterativo de Newton-Raphson (Huyakorn e Pinder, 1983 [14]).

4.3 Ilustração do acoplamento termo-hidráulico.

5.1 Visão geral dos acoplamentos (os acoplamentos realizados neste trabalho estão em vermelho e itálico).

5.2 Fluxograma reduzido do programa TRANSBEQC (esta Tese).

5.3 Interface do TRANSBEQC. 76

5.4 Malha de elementos finitos. 77

5.5 Visualização dos resultados.

5.6 llustração dos cenários. 80

5.7 Modelo conceitual (adaptado por Sturm e Morgan, 1981). 81

5.8 Modelo conceitual (adaptado por Sturm e Morgan, 1981). 81

5.9 Grupo dos NAPL's. $\quad 82$

5.10 Modelo Conceitual do DNAPL (Bedient et al., 1994 [52]). 83

5.11 Modelo Conceitual do LNAPL (Bedient et al., 1994 [52]). 83

6.1 Ilustração do cenário simulado no programa MINTRAN e TRANSBEQC. 85

6.2 Malha com o resultado do $\mathrm{pH}$ num dado passo de tempo. 86

6.3 Período de simulação = 1 ano 87 
6.4 Período de simulação = 10 anos 87

6.5 Período de simulação = 24 anos 87

6.6 Escala de cores 87

6.7 Comparação entre os valores de $\mathrm{pH}$ obtidos pelo MINTRAN e desta Tese. $\quad 87$

6.8 Comparação entre os valores de concentração de Fe (II) obtidos pelo MINTRAN e TRANSBEQC. 88

6.9 Ilustração do problema de difusão 1-D. 90

6.10 Malha de elementos finitos do problema de difusão 1-D. $\quad 90$

6.11 Comparação entre os valores normalizados de concentração de $\mathrm{O}_{2}$ obtidos pelo MINTOX e esta TESE. 90

6.12 Valores de variação do raio mineral ao longo dos anos. 91

6.13 llustração de uma coluna com infiltração de água na superfície e aquecimento na base. $\quad 92$

6.14 Malha do ensaio de uma coluna com infiltração de água na superfície e aquecimento na base. 93

6.15 Variação do fluxo de ar decorrente da Temperatura no contorno (fluxo isotérmico). 93

6.16 Variação do fluxo de ar decorrente da Temperatura no contorno (fluxo não isotérmico). 93

6.17 Variação da temperatura com a profundidade. 94

6.18 llustração do ensaio de injeção de água (embebição) e injeção de ar (secagem). 95

6.19 Malha do ensaio de injeção de água (embebição) e injeção de ar (secagem). 95

6.20 Curva característica do solo (Curvas de embebição e secagem). $\quad 95$

6.21 Dados da coluna. $\quad 97$

6.22 Malha referente à coluna do exemplo. 97

6.23 Variação da concentração de cálcio ao longo da coluna com o tempo. $\quad 98$

6.24 Variação da concentração de ferro (II) ao longo da coluna com o tempo. $\quad 98$

6.25 Variação da concentração de sulfato ao longo da coluna com o tempo. $\quad 99$

6.26 Variação do $p H$ ao longo da coluna com o tempo. 99

6.27 Formação do gesso numa coluna. $\quad 100$

6.28 Isoterma de adsorção do Zinco para vários valores de pH. 101 
6.29 Variação da Concentração do Zinco adsorvido e em solução para diferentes valores de $\mathrm{pH}$.

6.30 llustração do problema para avaliar a variação da temperatura na fonte em função das reações exotérmicas.

6.31 Malha de elementos finitos do problema para avaliar a variação da temperatura na fonte em função das reações exotérmicas.

6.32 Variação da temperatura na fonte em função das reações exotérmicas, logo abaixo da fonte (superfície do terreno).

6.33 Isotermas para o caso de existência de reações exotérmicas na fonte.

6.34 Comparação entre a variação do fluxo de calor para os casos com e sem reações exotérmicas na fonte.

6.35 Coluna usada no ensaio para verificação da influência da temperatura no fluxo bifásico.

6.36 Malha da coluna usada no ensaio para verificação da influência da temperatura no fluxo bifásico.

6.37 Variação da saturação decorrente da variação de temperatura no contorno.

6.38 Planta da Mina de Doyon (Sracek, 2004 [86]).

6.39 Modelo conceitual dos processos de transporte da mina de Doyon (Sracek, 2004 [86]).

6.40 Dados de entrada e condições de contorno da simulação da pilha.

6.41 Malha de elementos finitos usada na simulação da pilha. $\quad 108$

6.42 Período de simulação $=12$ meses 109

6.43 Período de simulação $=60$ meses $\quad 109$

6.44 Período de simulação $=162$ meses $\quad 109$

6.45 Escala de cores 109

6.46 Variação da temperatura na pilha. 110

6.47 Comparação da variação da temperatura na pilha ao longo de uma seção longitudinal (Tese x TOUGH2). 110

6.48 Vetores referentes ao fluxo de vapor no talude da pilha de estéril.

6.49 Comparação da variação da saturação na pilha ao longo de uma seção transversal (Tese x TOUGH2). 


\section{Lista de Tabelas}

3.1 Alguns programas e os respectivos modelos de biodegradação.

5.1 Tarefas realizadas.

5.2 Principais características de alguns programas de transporte de contaminantes disponíveis na literatura.

5.3 Características do cenário da barragem de rejeito e da pilha de estéril.

6.1 Concentração na fonte dos componentes na fase líquida. 86

6.2 Concentrações da fase mineral inicial. 86

6.3 Parâmetros de difusão para simulação 1-D. 89

6.4 Dados para construção da curva característica. 96 


\section{Lista de Símbolos}

$[B]$ - matriz de derivadas das funções de interpolação

$B_{f}$ - fator volume de formação da fase $f$

$B_{i}$ - termo convectivo na equação de transporte (velocidade)

$b_{m}$ - taxa de decaimento microbial

$C_{a e}$ - concentração do aceptor de elétrons na solução

$C_{f}$ - concentração de soluto na fase $f$

$C_{f k}$ - concentração do componente em cada fase

$C_{k}^{\text {int }}$ - valor intermediário de concentração

$C_{k}^{\text {phys }}$ - concentração no final do passo físico

$C_{l}$ - concentração de um constituinte na fase líquida

$C_{o}$ - concentração de um constituinte na fase NAPL

$C_{o c}$ - concentração de carbono orgânico natural

$C_{p a}$ - capacidade de calor específico do ar seco do solo

$C_{p l}$ - capacidade de calor específico da água do solo

$C_{p s}$ - capacidade de calor específico dos sólidos no solo

$C_{p v}$ - capacidade de calor específico do vapor do solo

$C_{N}$ - concentração da substância no NAPL

$C_{s}$ - concentração de contaminante na fase sólida

$C_{s f}^{m}$ - grau de solubilidade do constituinte $m$ na água

$C_{v}$ - concentração de um constituinte na fase gasosa

$C_{w}$ - concentração da substância na água

$c_{w}$ - capacidade térmica volumétrica da fase líquida

$c(\theta)$ - capacidade térmica volumétrica do meio poroso

$D$ - coeficiente de difusão da substância

Datm - difusividade molecular para o vapor através do ar

$D 1(x)$ - coeficiente de difusão do meio poroso

$D_{f}$ - coeficiente de difusão molecular aparente

$D_{h}$ - Difusividade isotérmica

$D_{i j}$ - Tensor do coeficiente de difusão efetivo da matriz de solo do $O_{2}$ na fase gasosa

$D_{i j f}$ - tensor de coeficientes de dispersão hidrodinâmica na fase $f$ 
$D_{i j l}$ - tensor de coeficientes de dispersão hidrodinâmica na fase líquida

$D_{i j o}$ - tensor de coeficientes de dispersão hidrodinâmica na fase líquida do NAPL

$D_{i j v}$ - tensor de coeficientes de dispersão hidrodinâmica na fase gasosa

$[D T]$ - matriz de difusividades térmicas no ar e no vapor

$D_{T a}$ - Difusividade térmica de "molhagem"

$D_{T v}$ - Difusividade térmica de vapor

$E_{i i}$ - termo dispersivo na equação de transporte (dispersividade)

$F$ - razão entre o consumo de elétron aceptor e o do contaminante

$F R$ - fator de retardamento

$F S$ - fluxo de massa de soluto por unidade de área por unidade de tempo

$g$ - aceleração da gravidade

$h$ - umidade relativa

$H_{c}$ - capacidade de calor específico do solo

$h_{n}$ - coeficientes desconhecidos representando a solução da equação nos pontos nodais

$j$ - nível de tempo anterior

$j+1$ - nível de tempo corrente em que a solução está sendo considerada

$J_{c a_{i}}$ - fluxo de $O_{2}$ causado pela convecção na fase gasosa

$J_{d a_{i}}$ - fluxo de $\mathrm{O}_{2}$ causado pela difusão na fase gasosa

$J_{c w_{i}}$ - fluxo de $\mathrm{O}_{2}$ causado pela convecção na fase dissolvida

$J_{d w_{i}}$ - fluxo de $\mathrm{O}_{2}$ causado pela difusão na fase dissolvida

$K$ - permeabilidade

$[K]$ - tensor de permeabilidades absolutas com influência dos coeficientes térmicos $\left(D_{h}, D_{T V}\right.$ e $\left.D_{T a}\right)$

$k_{o c}$ - taxa de decaimento de $1^{\mathrm{a}}$ ordem do carbono orgânico natural

$K_{a e}$ - constante de meia saturação do aceptor de elétrons

$K_{c}$ - constante de meia saturação do contaminante

$K_{d}$ - coeficiente de distribuição

$K_{D}$ - coeficiente de partição solo-água

$K_{f}$ - função que descreve a sorção do soluto no solo (isoterma de sorção)

$K_{H}$ - constante da lei de Henry

$K_{i j}$ - tensor de permeabilidades absolutas do solo

$K_{L}$ - coeficiente de partição água óleo

$k_{r f}$ - permeabilidade relativa do solo para a fase $f$

$k_{r w}$ - permeabilidade relativa do solo para água

$K_{t}$ - coeficiente de transferência de massa

$L$ - calor latente de vaporização da água do solo

$L L$ - operador diferencial espacial 
$M_{t}$ - concentração microbial total

$[N]$ - matriz das funções de interpolação

$n$ - porosidade

$n_{a r}$ - porosidade preenchida pelo ar

$N_{c}$ - número de componentes presentes em cada fase

$N D$ - ganho de massa por dissolução

$n_{\text {saturada }}$ - porosidade saturada com água

NumPN - número total de pontos nodais

$O_{a}$ - concentração volumétrica de $O_{2}$ na fase gasosa

$O_{T}$ - concentração volumétrica total de $O_{2}$

$P$ - Produção de $\mathrm{O}_{2}$

$p_{f}$ - pressão da fase $f$

$p_{n / w}$ - pressão capilar entre as duas fases

$p_{v}$ - pressão parcial de vapor no meio poroso

$\left\{p_{w}^{t+\Delta t}\right\}$ - vetor nodal da pressão da fase molhante no passo atual

$Q$ - fluxo total de calor

$q$ - remoção ou consumo de $O_{2}$ dissolvido do solo pela oxidação biológica (ação de bactérias) ou oxidação química

$Q_{f}$ - fonte da fase $f$

$[Q]_{j+\epsilon}$ - matriz de coeficientes

$\left\{q_{n}\right\}$ - vetor nodal da vazão prescrita da fase não molhante

$\left\{q_{w}\right\}$ - vetor nodal da vazão prescrita da fase molhante

$Q_{w}$ - fonte

$q(x, t)$ - termo de armazenamento devido consumo de oxigênio pelas partículas

$R$ - constante específica do gás

$r$ - taxa de destruição da pirita em $\mathrm{mol} / \mathrm{m}^{2} \mathrm{~s}$

$R_{k}^{\text {equil }}$ - massa relativa $(M / M)$ gerada ou consumida instantaneamente no equilíbrio do sistema no tempo $t+\Delta t^{\text {equil }}$

$R_{s}$ - razão de solubilidade do gás no líquido

$r r$ - contador de iteração com valor inicial igual a 1

$\frac{r}{v_{c}}$ - inclinação da linha formada em um gráfico monolog da concentração de contaminante versus o gradiente de distância ao longo da trajetória de fluxo

$s$ e $s+1$ - níveis de tempo anterior e atual, respectivamente

$S_{f}$ - grau de saturação da fase $f$

$S_{w}$ - grau de saturação da água

$\left\{S_{w}^{t}\right\}$ - vetor nodal do grau de saturação da fase molhante no passo anterior $\left\{S_{w}^{t+\Delta t}\right\}$ - vetor nodal do grau de saturação da fase molhante no passo atual $T$ - temperatura 
$T_{0}$ - temperatura de referência

temp - temperatura em Kelvin

$t_{c}$ - tempo a partir do qual a pressão de ar se iguala a $102 k P a$ no topo

$\left\{T_{t}\right\}$ - vetor nodal de tempetraturas determinado pela solução da equação de calor

$U_{A}^{*}$ - concentração de oxigênio no meio poroso, o asterisco indica que a variável é dimensional

$v_{c}$ - velocidade do contaminante retardada na direção $\mathrm{x}$

$V_{d g s}$ - volume de gás medido nas condições padrão $\left(V_{f}\right)$, dissolvido nas condições de pressão em questão

$V_{f}$ - volume da fase $f$ medido nas condições de pressão em questão

$\left|\bar{v}_{f}\right|$ - valor absoluto da velocidade de percolação

$V_{f s}$ - volume medido nas condições padrão (atmosféricas)

$v_{i f}$ - velocidade do fluxo da fase $f$ na direção $i$, calculada pela lei de Darcy

$\bar{v}_{i f}$ - velocidade de percolação

$v_{i g}$ - velocidade na direção $i$ das fases gasosa

$v_{i l}$ - velocidade do fluxo da fase líquida na direção $i$, calculada pela lei de Darcy

$v_{i o}$ - velocidade do fluxo da fase líquida do NAPL na direção $i$, calculada pela lei de Darcy

$v_{i v}$ - velocidade do fluxo da fase gasosa na direção $i$, calculada pela lei de Darcy

$V_{l s}$ - volume unitário de líquido contido no solo $\left(V_{f}\right)$

$X$ - parâmetro qualquer

$x_{1}, x_{2}, \ldots, x_{n}$ - váriaveis desconhecidas

$Y$ - coeficiente de produção microbial

$z$ - coordenada vertical

$\alpha$ - fator de ponderação no tempo $(\alpha \in[0,1])$

$\alpha_{l f}$ - coeficientes de mistura mecânica longitudinal

$\alpha_{t f}$ - coeficientes de mistura mecânica transversal

$\alpha_{x}$ - dispersividade

$\delta_{i j}$ - delta de Kronecker

$\Delta t$ - incremento de tempo

$\delta\left(t+\frac{\Delta t}{2}\right)$ - função delta de Dirac $(1 / T)$

$\Delta x_{i}$ - comprimento característico de um elemento finito

$\Delta x_{J}^{r+1}$ - vetor deslocamento

$\Delta t_{j}$ - variação do tempo

$\varepsilon$ - tolerância prescrita para valores de $x$

$\eta$ - teor volumétrico de vapor 
$\Gamma_{e}$ - segmento do contorno do elemento $e$

$\gamma_{l}$ - densidade do líquido

$\lambda$ - coeficiente de condutividade térmica do solo

$\lambda_{b}$ - taxa de decaimento biológico de $1^{\mathrm{a}}$ ordem

$\lambda_{d}$ - taxa de decaimento de $1^{\mathrm{a}}$ ordem na unidade de $t^{-1}$

$\lambda_{i j}$ - coeficiente de condutividade termal aparente do solo

$\lambda^{m}$ - coeficiente de transferência de massa do constituinte $m$ que incorpora a área interfacial

$\mu_{f}$ - viscosidade cinemática da fase $f$

$\mu_{l}$ - constante de $1^{\mathrm{a}}$ ordem para degradação na fase líquida

$\mu_{\max }$ - taxa de utilização máxima da concentração por unidade de massa de microorganismos

$\mu_{o}$ - constante de $1^{\mathrm{a}}$ ordem para degradação na fase NAPL

$\mu_{s}$ - taxa constante de $1^{\mathrm{a}}$ ordem para degradação na fase sólida

$\mu_{v}$ - constante de $1^{\mathrm{a}}$ ordem para degradação na fase gasosa

$\mu_{w}$ - viscosidade cinemática da água

$\nu_{v}$ - fator de fluxo de massa

$\Omega_{e}$ - domínio ocupado pelo elemento $e$

$\omega_{s}$ - índice de performance

$\Phi$ - teor de calor do solo

$\phi$ - potencial hidráulico

$\phi_{f}$ - potencial hidráulico da fase $f$

$\phi_{g}$ - função potencial da fase gasosa

$\phi_{l}$ - função potencial da fase líquida

$\phi_{N}$ - teor volumétrico de NAPL

$\phi_{n}$ - funções lineares básicas que satisfazem a condição $\phi_{n}\left(x_{m}, z_{m}\right)=\delta_{m n}$

$\Psi$ - carga de pressão

$\rho$ - densidade da fase sólida

$\rho_{0}$ - massa específica do vapor d'água saturado

$\rho_{d}$ - massa específica seca do solo

$\rho_{f}$ - massa específica da fase $f$

$\rho_{s}$ - densidade dos sólidos do solo

$\tau_{v}$ - fator de tortuosidade para difusão de gases nos solos

$\theta$ - umidade volumétrica

$\theta_{a}$ - umidade volumétrica do ar

$\theta_{g}$ - umidade volumétrica do ar no solo

subscritos:

$f$ - fase do fluido

$g$ - fase gasosa 
$l$ - fase líquida

$m$ - constituinte químico

$N$ - fase NAPL

$n$ - fase mineral

$o$ - matéria orgânica

$w$ - fase líquida (água)

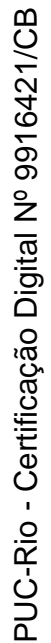

